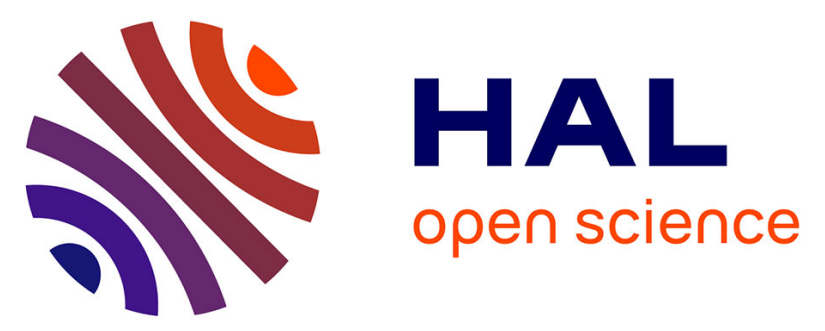

\title{
Relations entre l'état angulaire d'une vapeur atomique soumise au pompage optique et ses propriétés d'absorption et de dispersion. - Première Partie
}

\author{
F. Laloë, M. Leduc, P. Minguzzi
}

\section{- To cite this version:}

F. Laloë, M. Leduc, P. Minguzzi. Relations entre l'état angulaire d'une vapeur atomique soumise au pompage optique et ses propriétés d'absorption et de dispersion. - Première Partie. Journal de Physique, 1969, 30 (2-3), pp.277-288. 10.1051/jphys:01969003002-3027700 . jpa-00206785

\section{HAL Id: jpa-00206785 https://hal.science/jpa-00206785}

Submitted on 1 Jan 1969

HAL is a multi-disciplinary open access archive for the deposit and dissemination of scientific research documents, whether they are published or not. The documents may come from teaching and research institutions in France or abroad, or from public or private research centers.
L'archive ouverte pluridisciplinaire HAL, est destinée au dépôt et à la diffusion de documents scientifiques de niveau recherche, publiés ou non, émanant des établissements d'enseignement et de recherche français ou étrangers, des laboratoires publics ou privés. 


\title{
RELATIONS ENTRE L'ÉTAT ANGULAIRE D'UNE VAPEUR ATOMIQUE SOUMISE AU POMPAGE OPTIQUE ET SES PROPRIÉTÉS D'ABSORPTION ET DE DISPERSION
}

\author{
Première Partie ( $\left.{ }^{1}\right)$
}

\author{
Par F. LALOË, M. LEDUG et P. MINGUZZI (2), \\ Faculté des Sciences de Paris, Laboratoire de Spectroscopie Hertzienne de l'E.N.S., associé au G.N.R.S.
}

(Reçu le 31 octobre 1968.)

\begin{abstract}
Résumé. - Cet article est consacré à l'étude de l'optimisation des signaux de détection optique obtenus dans une expérience de pompage optique. Les formules générales obtenues dans des publications antérieures sont explicitées en fonction des divers paramètres intervenant dans une expérience : direction de propagation du faisceau lumineux, composition spectrale, nature du polariseur et de l'analyseur; on peut ainsi déterminer dans chaque cas particulier le montage qui permet de détecter dans les meilleures conditions les observables atomiques auxquelles on s'intéresse.

Les calculs exposés font intervenir le formalisme des opérateurs tensoriels irréductibles. Dans un second article, nous montrerons comment les résultats obtenus de cette manière peuvent être établis à partir de considérations générales purement géométriques.

Abstract. - In the present paper, we study how to optimize the signals of optical detection obtained in an optical pumping experiment. The general formulae established in earlier papers are developed in terms of various parameters : direction of propagation of the light beam, its spectral repartition and nature of polarizer and analyser. One then determines in each case the best experimental set up for detecting the selected atomic observable.

The calculations presented here make use of the formalism of irreducible tensorial operators. In a second paper to follow soon, we will show how the results thus obtained can be established through general geometrical considerations.
\end{abstract}

Introduction. - Dans une expérience de pompage optique, il est possible d'utiliser un faisceau lumineux auxiliaire pour détecter l'évolution dans l'état fondamental de certaines grandeurs atomiques. On sait que deux cas sont possibles :

Dans le premier cas, la composition spectrale du faisceau est telle que la fréquence de certaines vibrations lumineuses correspond à l'une des transitions optiques des atomes étudiés (faisceau résonnant). La vapeur atomique se comporte alors comme un milieu absorbant et peut notamment présenter un dichroïsme qui dépend de l'orientation ou de l'alignement des atomes dans l'état fondamental ; la mesure de l'absorption par la vapeur d'un faisceau lumineux polarisé permet donc de détecter ces observables. De nombreuses expériences de ce type ont été réalisées, par exemple dans le cas du mercure ([1] à [3]), des alcalins ([4] à [10]), ou encore de l'hélium ([11] à [15]).

(1) La seconde partie de cette étude paraîtra dans le prochain numéro du Journal de Physique.

(2) Istituto di Fisica dell'Università, Pisa, Gruppo Nazionale di Struttura della Materia del C.N.R.
On sait d'ailleurs qu'il existe un autre aspect, complémentaire de celui qui vient d'être décrit, de l'interaction entre les atomes et un faisceau lumineux résonnant; sous l'influence de ce dernier, les atomes effectuent des transitions réelles entre l'état fondamental et l'état excité, de sorte que leur répartition entre les divers sous-niveaux de l'état fondamental peut se trouver profondément modifiée : c'est le principe du pompage optique [16], dont Barrat et Cohen-Tannoudji ont donné une description théorique très complète, notamment dans deux articles publiés au Journal de Physique [17] que nous désignerons par JP1 et JP2.

Dans le deuxième cas ${ }^{(3)}$, le faisceau lumineux

$\left.{ }^{3}\right)$ Les deux cas envisagés correspondent à deux cas limites; en effet, en modifiant la composition spectrale du faisceau lumineux détecteur, on passe continûment d'un faisceau résonnant à un faisceau non résonnant. En pratique, le faisceau induit généralement dans la vapeur à la fois des transitions réelles et virtuelles; il se trouve toutefois que dans certains cas, comme celui du mercure, on peut utiliser la structure isotopique de la raie optique de résonance pour séparer assez bien les deux cas. 
détecteur ne contient aucune fréquence qui corresponde exactement à une transition atomique (faisceau non résonnant). Il ne peut donc être absorbé par la vapeur, et ne fait subir aux atomes que des transitions virtuelles. Seules peuvent alors être utilisées les propriétés de dispersion de la vapeur; on détecte dans ce cas sa biréfringence en mesurant, au moyen d'un analyseur, la modification de polarisation que subit un faisceau lumineux lors de sa traversée. L'application de cette méthode aux expériences de pompage optique, proposée en premier par Gozzini [18], a été réalisée en utilisant diverses vapeurs atomiques ([19] à [24]).

Cohen-Tannoudji et Laloë ont établi un formalisme qui permet dans tous les cas de calculer la modification que subit la matrice polarisation d'un faisceau lumineux détecteur lorsqu'il traverse une vapeur atomique soumise au pompage optique. Ce travail a fait l'objet de deux publications au Journal de Physique [25] que nous désignerons par JP3 et JP4. Les équations données dans ces articles sont très générales; toutefois, les signaux de détection optique ne sont pas explicitement calculés. Or, en pratique, il est utile de connaître leur expression détaillée, de façon à mettre en évidence l'influence sur les signaux obtenus des différents paramètres qui caractérisent un faisceau détecteur (direction de propagation, polariseur, analyseur, isotope contenu dans la lampe) : il est alors possible de choisir dans chaque cas le montage qui permet d'observer dans les meilleures conditions les grandeurs atomiques auxquelles on s'intéresse.

Ce premier article est donc consacré à l'étude détaillée du signal de détection optique; pour simplifier, nous nous sommes limités au cas des épaisseurs optiques faibles et de l'effet paramagnétique. Divers problèmes pratiques qui peuvent se poser dans une expérience de pompage optique utilisant un faisceau détecteur sont traités : détermination du polariseur et de l'analyseur les plus appropriés pour détecter chaque grandeur atomique, avec un faisceau lumineux résonnant ou non; connaissance des diverses modulations lumineuses qui peuvent être observées lorsque l'on effectue une résonance magnétique dans l'état fondamental, et que le faisceau lumineux se propage soit parallèlement au champ magnétique statique, soit perpendiculairement (méthode du « faisceau croisé »); influence de la composition spectrale du faisceau détecteur lorsque la raie de résonance optique utilisée comprend plusieurs composantes (les exemples pratiques étudiés sont ceux de la structure hyperfine de la raie de résonance des isotopes impairs du mercure, et de la structure fine de la raie $\lambda=1,1 \mu$ de ${ }^{4} \mathrm{He}$; le cas des alcalins est traité en appendice).

La seconde partie de ce travail paraîtra dans le prochain numéro du Journal de Physique; elle a pour objet d'étudier le lien qui existe entre les propriétés d'anisotropie optique de la vapeur et l'état angulaire des atomes dans l'état fondamental. On montre en effet dans JP4 que, dans le cas de l'effet paramagné- tique, le dichroïsme ou la biréfringence de la vapeur sont entièrement liés aux propriétés de la matrice densité $\sigma_{f}(t)$ des atomes; par exemple, tous deux sont nuls lorsque $\sigma_{f}(t)$ est invariante par rotation. Dans cet article, nous donnerons une représentation géométrique des diverses propriétés de symétrie de $\sigma_{f}(t)$ : nous caractériserons l'orientation dans l'état fondamental par un vecteur, l'alignement par un ellipsoïde. Nous montrerons comment il est possible de déterminer de manière purement géométrique les diverses propriétés d'anisotropie optique de la vapeur (caractérisée par ses « polarisations principales » et ses « indices principaux »), lorsque la direction de propagation du faisceau lumineux détecteur est quelconque.

Signalons enfin que diverses expériences portant sur ${ }^{201} \mathrm{Hg}$ nous ont permis de vérifier de manière satisfaisante un certain nombre des résultats qui vont être exposés; la description de ces expériences a été publiée par ailleurs [26].

\section{ETUDE PRATIQUE DES CONDITIONS OPTIMALES DE DETECTION OPTIQUE}

A. Rappels et remarques prélíminaires. Calcul du signal de détection optique. - 1 . НYротнÈSES ET NOTATIONS. ÉQUATIONS DE DÉPART. - Nous appelons $|I, \mu\rangle$ les divers sous-niveaux Zeeman de l'état fondamental, d'énergies $\mu \hbar \omega_{f} ;|F, m\rangle$ les divers sous-niveaux Zeeman de l'état excité, d'énergies $\hbar\left(\omega_{0}+m \omega_{\mathrm{e}}\right)$. $\Delta$ et $\Delta^{\prime}$ désignent respectivement la largeur spectrale du faisceau détecteur et la largeur Doppler de la raie d'absorption de la vapeur. Nous faisons les mêmes hypothèses qu'au paragraphe III de JP4; nous supposons notamment que l'épaisseur optique de la vapeur est faible, et que l'effet paramagnétique intervient seul $\left(^{4}\right)$ (c'est-à-dire que l'on a $\omega_{e, f} \ll \Delta$ ou $\Delta^{\prime}$ ).

Dans ces conditions, on montre dans JP4 que la différence entre la matrice polarisation $\pi_{T}$ du faisceau lumineux transmis et la matrice polarisation $\pi_{I}$ du faisceau incident est donnée par :

$\pi_{T^{\prime}}-\pi_{I}=-\alpha \frac{\Gamma^{\prime}}{2}\left[B(t), \pi_{I}\right]_{+}-i \alpha \Delta E^{\prime}\left[B(t), \pi_{I}\right]$.

Dans cette expression, $\alpha$ est une constante, proportionnelle à l'épaisseur optique de la cellule, et inversement proportionnelle à l'intensité lumineuse incidente $I_{0}$. La matrice $B(t)$ est une matrice à deux dimensions, qui dépend de $\sigma_{f}(t)$ et de la direction $\mathrm{OZ}$ de propagation du faisceau lumineux; elle agit dans l'espace $\mathscr{F}_{X Y}^{(2)}$ des vecteurs polarisation lumineuse contenus dans le plan XOY (OXYZ est un trièdre trirectangle direct). $B(t)$ est définie par :

$\left\langle\mathbf{e}_{i}|B(t)| \mathbf{e}_{j}\right\rangle=\operatorname{Tr}_{f}\left\{\sigma_{f}(t)\left(\mathbf{e}_{i}^{*}\right.\right.$. D $\left.) P_{e}\left(\mathbf{e}_{j} . \mathbf{D}\right)\right\}$

(4) Dans le paragraphe D et dans l'appendice I, nous tiendrons toutefois compte de différences d'énergie à l'intérieur de l'état excité ou fondamental, et donc de l'effet diamagnétique. 
où $P_{e}$ est le projecteur sur les états excités considérés $\left(^{5}\right)$, et $\mathbf{D}$ la « partie angulaire » de l'opérateur dipôle électrique.

$\Gamma^{\prime}$ et $\Delta E^{\prime}$ sont les quantités déjà introduites dans JP1, JP2 et JP4, et dont la définition s'écrit, avec les notations de ces articles :

$$
\begin{aligned}
& \frac{\Gamma^{\prime}}{2}+i \Delta E^{\prime}=\frac{i q^{2}}{\hbar^{2}} \frac{|\langle F\|\mathbf{S}\| I\rangle|^{2}}{2 F+1} \\
& \int I(\omega) \mathrm{d} \omega \int \frac{f(\mathbf{v}) \mathrm{d}^{3} \mathbf{v}}{\omega-\omega_{0}-\mathbf{k} \cdot \mathbf{v}+i \Gamma / 2}
\end{aligned}
$$

$\Gamma^{\prime}$ et $\Delta E^{\prime}$ correspondent respectivement à l'élargissement et au déplacement de l'état fondamental dus à l'interaction des atomes avec le faisceau lumineux détecteur. Dans l'équation (I.A.1), le terme qui contient $\Gamma^{\prime}$ correspond à l'influence sur les polarisations lumineuses de l'absorption de la vapeur atomique; le terme qui contient $\Delta E^{\prime}$ correspond à l'influence de la dispersion

L'expression (I.A.1) permet de calculer les signaux de détection optique obtenus dans chaque expérience; nous allons envisager successivement divers cas possibles.

2. Classement des différents types D'expérience. - a) Détection optique sans polariseur ni analyseur. — On suppose qu'aucun polariseur ni analyseur n'est placé sur le trajet du faisceau lumineux détecteur, et on appelle $I_{0}+\Delta I$ l'intensité lumineuse transmise par la cellule et recueillie par le photomultiplicateur. En utilisant (I.A.1), on voit que $\Delta I$ est donné par :

$$
\Delta I=-\left(\alpha \Gamma^{\prime} / 2\right) I_{0} \operatorname{Tr}\{B(t)\} .
$$

Seules interviennent les propriétés d'absorption de la vapeur. On montre dans JP4 que $\operatorname{Tr}\{B(t)\}$ ne peut dépendre que de l'alignement des atomes dans la direction de propagation du faisceau lumineux. Les signaux de détection optique sont donc connus dans ce cas, sur lequel nous ne reviendrons pas.

b) Détection optique avec un polariseur seul, ou un analyseur seul. - Si l'on place sur le trajet du faisceau lumineux un polariseur, correspondant à un vecteur polarisation $\left|\mathbf{e}_{\lambda_{0}}\right\rangle$, mais pas d'analyseur, on a :

$$
\pi_{I}=I_{0}\left|\mathbf{e}_{\lambda_{0}}\right\rangle\left\langle\mathbf{e}_{\lambda_{0}}\right| \text {. }
$$

Le photomultiplicateur reçoit une intensité lumineuse $I_{0}+\Delta I$, où $\Delta I$ est donné par :

$$
\begin{aligned}
\Delta I=-\alpha \Gamma^{\prime} \operatorname{Tr} & \left\{\pi_{I} B(t)\right\} \\
& =-\alpha \Gamma^{\prime} I_{0}\left\langle\mathbf{e}_{\lambda_{0}}|B(t)| \mathbf{e}_{\lambda_{0}}\right\rangle .
\end{aligned}
$$

Dans ce cas encore, seules interviennent les propriétés d'absorption de la vapeur.

$\left(^{5}\right) P_{\mathrm{e}}$ est donc, avec les hypothèses que nous avons faites, le projecteur sur le niveau $F$ :

$$
P_{e}=\sum_{m}|F, m\rangle\langle F, m ! \text {. }
$$

Le cas où l'on n'utilise aucun polariseur, mais un analyseur seul correspondant à la polarisation $\left|\mathbf{e}_{a}\right\rangle$ (cf. JP3, § I.6, d), se ramène immédiatement à celui qui vient d'être traité. En effet, on voit sur l'équation (I.A.1) que le photomultiplicateur reçoit alors une intensité lumineuse égale à :

$$
\left(I_{0} / 2\right)\left[1-\alpha \Gamma^{\prime}\left\langle\mathbf{e}_{a}|B(t)| \mathbf{e}_{a}\right\rangle\right] .
$$

Il suffit donc de faire $\left|\mathbf{e}_{a}\right\rangle=\left|\mathbf{e}_{\lambda_{0}}\right\rangle$ pour être ramené au cas précédent.

c) Détection optique avec un polariseur et un analyseur. - La mesure la plus générale consiste à placer à la fois un polariseur et un analyseur sur le trajet du faisceau lumineux. Dans ce cas, le photomultiplicateur reçoit une intensité lumineuse $\left\langle\mathbf{e}_{a}\left|\pi_{I}\right| \mathbf{e}_{a}\right\rangle+\Delta I$, avec :

$$
\begin{aligned}
\Delta I= & -\alpha I_{0}\left(\Gamma^{\prime} / 2\right)\left\{\left\langle\mathbf{e}_{a}|B(t)| \mathbf{e}_{\lambda_{0}}\right\rangle\left\langle\mathbf{e}_{\lambda_{0}} \mid \mathbf{e}_{a}\right\rangle+\text { c. c. }\right\} \\
& -i \alpha I_{0} \Delta E^{\prime}\left\{\left\langle\mathbf{e}_{a}|B(t)| \mathbf{e}_{\lambda_{0}}\right\rangle\left\langle\mathbf{e}_{\lambda_{0}} \mid \mathbf{e}_{a}\right\rangle-\text { c. c. }\right\} .
\end{aligned}
$$

Cette formule permet de faire quelques remarques :

a) Les variations du signal de détection optique en fonction de $\sigma_{f}(t),\left|\mathbf{e}_{\lambda_{0}}\right\rangle,\left|\mathbf{e}_{a}\right\rangle$, sont contenues dans le facteur $\left\langle\mathbf{e}_{a}|B(t)| \mathbf{e}_{\lambda_{0}}\right\rangle\left\langle\mathbf{e}_{\lambda_{0}} \mid \mathbf{e}_{a}\right\rangle$. La partie réelle de ce facteur permet de connaître les signaux optiques correspondant aux transitions réelles; la partie imaginaire, ceux qui correspondent aux transitions virtuelles.

$\beta)$ Si l'on intervertit $\left|\mathbf{e}_{\lambda_{0}}\right\rangle$ et $\left|\mathbf{e}_{a}\right\rangle$, les signaux dus aux transitions réelles ne changent pas; ceux qui sont dus aux transitions virtuelles changent de signe.

$\gamma)$ Si le polariseur et l'analyseur correspondent à des polarisations orthogonales $\left(\left\langle\mathbf{e}_{\lambda_{0}} \mid \mathbf{e}_{a}\right\rangle=0\right)$, les signaux de détection optiques sont constamment nuls. Un tel montage ne peut être utilisé que dans les cas où l'épaisseur optique de la vapeur atomique n'est pas faible [21], [27].

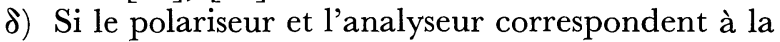
même polarisation, la comparaison de (I.A.6) et (I.A.8), où l'on a posé $\left|\mathbf{e}_{a}\right\rangle=\left|\mathbf{e}_{\lambda_{0}}\right\rangle$, montre que l'analyseur (ou le polariseur) ne joue aucun rôle dans le signal de détection optique. Il n'est donc pas nécessaire d'étudier à part les cas cités en b) (polariseur seul ou analyseur seul); on peut les considérer comme un cas particulier des signaux obtenus dans le cas général.

3. EXPRESSION DÉtAILlÉE DU SIGNAL DE DÉTEGTION OPTIQUE. - Les relations (III.13) et (III.14) de JP4 permettent de calculer les éléments de matrice de $B(t)$. En choisissant comme base les deux vecteurs $\left|\mathbf{e}_{X}\right\rangle$ et $\left|\mathbf{e}_{Y}\right\rangle$ portés par les axes OX et OY, nous obtenons :

$$
\begin{array}{r}
B(t)=\left(\begin{array}{cc}
b_{0}(t)+b_{3}(t) & b_{1}(t)-i b_{2}(t) \\
b_{1}(t)+i b_{2}(t) & b_{0}(t)-b_{3}(t)
\end{array}\right) \\
=\sum_{n=0}^{3} b_{n}(t) \sigma_{n}
\end{array}
$$


$\sigma_{0}$ est la matrice unité de dimension $2 ; \sigma_{1}, \sigma_{2}, \sigma_{3}$ sont les trois matrices de Pauli. Les quatre nombres réels $b_{n}(t)$ sont définis par :

$$
\left\{\begin{aligned}
b_{0}(t) & =-\frac{1}{\sqrt{6}}\left[\sqrt{2}\left\langle T_{0}^{(0)}\right\rangle+\left\langle T_{0}^{(2)}\right\rangle\right] \\
b_{1}(t) & =\frac{i}{2}\left[\left\langle T_{-2}^{(2)}\right\rangle-\left\langle T_{+2}^{(2)}\right\rangle\right] \\
b_{2}(t) & =\frac{1}{\sqrt{2}}\left\langle T_{0}^{(1)}\right\rangle \\
b_{3}(t) & =\frac{1}{2}\left[\left\langle T_{-2}^{(2)}\right\rangle+\left\langle T_{+2}^{(2)}\right\rangle\right]
\end{aligned}\right.
$$

avec :

$$
\left\langle T_{q}^{(k)}\right\rangle=\operatorname{Tr}_{f}\left\{\sigma_{f}(t) T_{q}^{(k)}\right\}
$$

Rappelons la définition et le sens physique des opérateurs $T^{(k)}$; leurs composantes standard dans les axes OXYZ sont données par :

$$
T_{q}^{(k)}=\sum_{q^{\prime}+q^{\prime \prime}=q}\left\langle 11 q^{\prime} q^{\prime \prime} \mid k q\right\rangle D_{q^{\prime}} P_{e} D_{q^{\prime \prime}}
$$

où $D_{q}$ et $D_{q^{\prime}}$ sont les composantes standard du vecteur $\mathbf{D}$ dans les mêmes axes.

$T_{0}^{(0)}$ est proportionnel à la population totale de l'état fondamental $\left(\left\langle T_{0}^{(0)}\right\rangle\right.$ est égal à une constante $\left.c^{(0)}\right) . T_{0}^{(1)}$ est proportionnel à $I_{Z}$, projection du moment cinétique de l'état fondamental sur la direction $\mathrm{OZ}$; on pose :

$$
\left\langle T_{0}^{(1)}\right\rangle=c^{(1)}\left\langle I_{Z}\right\rangle
$$

où $c^{(1)}$ est un coefficient réel. $T_{0}^{(2)}, T_{2}^{(2)}$ et $T_{-2}^{(2)}$ correspondent à l'alignement des atomes dans l'état fondamental; on introduit le coefficient réel $c^{(2)}$ en posant :

$\left\{\begin{array}{l}\left\langle T_{0}^{(2)}\right\rangle=\frac{1}{\sqrt{6}} c^{(2)}\left[3\left\langle I_{Z}^{2}\right\rangle-I(I+1)\right] \\ \left\langle T_{ \pm 2}^{(2)}\right\rangle=\frac{1}{2} c^{(2)}\left\langle\left(I_{X} \pm i I_{Y}\right)^{2}\right\rangle .\end{array}\right.$

Pour reporter l'expression (I.A.9) dans l'équation (I.A.8), il est commode d'introduire les nombres réels $R_{n}$ et $V_{n}$ définis par :

$R_{n}+i V_{n}=2\left\langle\mathbf{e}_{a}\left|\sigma_{n}\right| \mathbf{e}_{\lambda_{0}}\right\rangle\left\langle\mathbf{e}_{\lambda_{0}} \mid \mathbf{e}_{a}\right\rangle$.

On a alors :

$\Delta I=-\alpha I_{0} \sum_{n} b_{n}(t)\left[\frac{\Gamma^{\prime}}{2} R_{n}-\Delta E^{\prime} V_{n}\right]$

Cette équation va nous permettre d'étudier de manière détaillée les signaux de détection optique. Les différents paramètres qui interviennent dans le problème qui nous intéresse sont en effet séparés dans (I.A.15) : l'influence du polariseur et de l'analyseur est contenue dans les facteurs $R_{n}$ et $V_{n}$; celle des observables atomiques et de leur évolution en fonction du temps dans les nombres $b_{n}(t)$; quant à l'influence de la composition spectrale du faisceau détecteur, elle apparaît dans $\Gamma^{\prime}$ et $\Delta E^{\prime}$. Les trois paragraphes qui vont suivre seront consacrés à l'étude successive du rôle de ces différents paramètres.

B. Rôle du polariseur et de l'analyseur. - Nous nous proposons dans ce paragraphe de déterminer le polariseur et l'analyseur qui, placés sur le trajet du faisceau détecteur, rendent maximum le signal optique correspondant à une grandeur atomique donnée; de plus, nous chercherons à isoler cette grandeur, c'est-à-dire à annuler la contribution au signal des autres observables.

Nous supposons que le faisceau lumineux traverse dans l'ordre (voir fig. 1) : un polariseur P, formé d'un

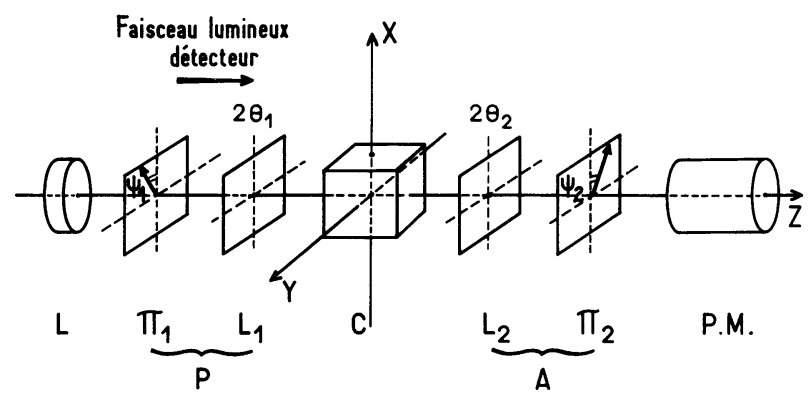

FIG. 1.

polariseur linéaire $\pi_{\mathbf{1}}$ transmettant les vibrations lumineuses qui font un angle $\Psi_{1}$ avec $\mathrm{OX}$, et d'une lame biréfringente $\mathrm{L}_{1}$ d'axes parallèles à $\mathrm{OX}$ (axe rapide) et OY (axe lent), et de retard $2 \theta_{1}$; la cellule contenant la vapeur atomique étudiée; un analyseur A formé d'une lame biréfringente $L_{2}$ d'axes parallèles à ceux de $\mathrm{L}_{1}$ et de retard $2 \theta_{2}$, et d'un polariseur linéaire transmettant les vibrations lumineuses qui font un angle $\Psi_{2}$ avec OX. Le faisceau lumineux est ensuite recueilli par un photomultiplicateur PM.

Dans ces conditions, les vecteurs $\left|\mathbf{e}_{\lambda_{0}}\right\rangle$ et $\left|\mathbf{e}_{a}\right\rangle$ s'écrivent :

$\left\{\begin{array}{l}\left|\mathbf{e}_{\lambda_{0}}\right\rangle=\mathrm{e}^{-i \theta_{1}} \cos \Psi_{\mathbf{1}}\left|\mathbf{e}_{x}\right\rangle+\mathrm{e}^{i \theta_{1}} \sin \Psi_{\mathbf{1}}\left|\mathbf{e}_{y}\right\rangle \\ \left|\mathbf{e}_{a}\right\rangle=\mathrm{e}^{i \theta_{2}} \cos \Psi_{2}\left|\mathbf{e}_{x}\right\rangle+\mathrm{e}^{-i \theta_{2}} \sin \Psi_{2}\left|\mathbf{e}_{y}\right\rangle .\end{array}\right.$

Ces expressions, qui correspondent aux polarisations les plus générales contenues dans le plan XOY, permettent de calculer les quantités $R_{n}$ et $V_{n}$; les valeurs obtenues sont portées sur le tableau I. Nous allons discuter les résultats de ce tableau en étudiant successivement le cas d'un faisceau résonnant et celui d'un faisceau non résonnant.

1. Faisceau Résonnant. - Pour un faisceau résonnant, on a $\Delta E^{\prime} \ll \Gamma^{\prime}$ et, dans l'expression (I.A.12), seuls interviennent les termes qui contiennent les nombres $R_{n}$. On voit sur le tableau I que l'on peut rendre 
chacun de ces quatre nombres maximum en posant $\Psi_{1}=\Psi_{2}$ et $\theta_{1}=-\theta_{2}$, c'est-à-dire $\left|\mathbf{e}_{\lambda_{0}}\right\rangle=\left|\mathbf{e}_{a}\right\rangle$. D'après le $\S \mathrm{A} .4, \mathrm{~d}$, ce résultat entraîne que l'analyseur (ou le polariseur) est inutile. On a alors :

$$
\begin{array}{llll}
R_{0}=2, & \text { indépendant de } & \Psi_{1} \text { et } \theta_{1} & \\
R_{1}=2 \sin 2 \Psi_{1} \cdot \cos 2 \theta_{1}, & \text { maximum si } & \Psi_{1}=\pi / 4, \theta_{1}=0 & \text { (polariseur linéaire) } \\
R_{2}=2 \sin 2 \Psi_{1} \cdot \sin 2 \theta_{1}, & \text { maximum si } & \Psi_{1}=\pi / 4, \theta_{1}=\pi / 4 & \text { (polariseur circulaire) } \\
R_{3}=2 \cos 2 \Psi_{1}, & \text { maximum si } & \Psi_{1}=0, \theta_{1} \text { quelconque } & \text { (polariseur linéaire) }
\end{array}
$$

On a résumé sur le tableau II les résultats obtenus, qui permettent de choisir les polariseurs les mieux adaptés à la détection optique des grandeurs $b_{n}(t)$ avec un faisceau résonnant. On voit que, dans tous les cas, on détecte avec un faisceau résonnant la grandeur $b_{0}(t)$ (c'est-à-dire l'alignement dans la direction $\mathrm{OZ}$ ).

Nous pouvons distinguer deux cas particuliers :

a) Avec un polariseur circulaire, on détecte, en plus de $b_{0}(t)$, la grandeur $b_{2}(t)=c^{(\mathbf{1})}\left\langle I_{Z}\right\rangle$ (pour isoler l'observable $I_{Z}$, il serait nécessaire de mesurer la différence des absorptions par la vapeur des deux polarisations circulaires droite et gauche). Nous retrouvons le fait que l'orientation dans l'état fondamental correspond au dichroïsme circulaire de la vapeur.

b) Avec un polariseur linéaire, on ne peut détecter que l'alignement, et le signal de détection optique s'écrit :

$\Delta I=-\alpha \Gamma^{\prime} I_{0}\left[b_{0}(t)+b_{1}(t) \sin 2 \Psi_{1}+b_{3}(t) \cos 2 \Psi_{1}^{\circ}\right]$.
Or, d'après les relations (I.A.13), on a :

$$
\left\{\begin{array}{l}
b_{1}(t)=(1 / 2) c^{(2)}\left\langle I_{X} I_{Y}+I_{Y} I_{X}\right\rangle \\
b_{3}(t)=(1 / 2) c^{(2)}\left\langle I_{X}^{2}-I_{Y}^{2}\right\rangle
\end{array}\right.
$$

L'étude de $\Delta I$ en fonction $\Psi_{1}$ permet donc de connaître ces deux composantes de l'alignement. Le fait que l'alignement des atomes intervient lorsque la polarisation incidente est linéaire montre bien qu'il est lié à un dichroïsme linéaire de la vapeur.

2. FAISCEAU NON RÉSONNANT. - Pour un faisceau non résonnant, on a $\Gamma^{\prime} \ll \Delta E^{\prime}$, et on ne conserve dans l'expression (I.A.15) que les termes qui contiennent les nombres $V_{n}$ (voir tableau $\mathrm{I}$ ).

$V_{0}$ est toujours nul : la quantité $b_{0}(t)$ n'intervient pas dans la biréfringence de la vapeur et ne peut se détecter avec un faisceau non résonnant.

$V_{1}$ est maximum si :

$$
\theta_{1}=\theta_{2}=\pi / 4 \text { et si } \Psi_{1}+\Psi_{2}=\pi / 4
$$

\begin{tabular}{|c|c|c|}
\hline$n$ & $R_{n}=\left\langle\mathbf{e}_{a}\left|\sigma_{n}\right| \mathbf{e}_{\lambda_{0}}\right\rangle\left\langle\mathbf{e}_{\lambda_{0}} \mid \mathbf{e}_{a}\right\rangle+$ c. c. & $V_{n}=-i\left(\left\langle\mathbf{e}_{a}\left|\sigma_{n}\right| \mathbf{e}_{\lambda_{0}}\right\rangle\left\langle\mathbf{e}_{\lambda_{0}} \mid \mathbf{e}_{a}\right\rangle-\right.$ c. c. $)$ \\
\hline 0 & $\begin{array}{r}2 \cos ^{2}\left(\theta_{1}+\theta_{2}\right) \cos ^{2}\left(\Psi_{1}-\Psi_{2}\right)+2 \sin ^{2}\left(\theta_{1}+\theta_{2}\right) \cos ^{2}\left(\Psi_{1}+\Psi_{2}\right) \\
=2\left|\left\langle\mathbf{e}_{\lambda_{0}} \mid \mathbf{e}_{a}\right\rangle\right|^{2}\end{array}$ & 0 \\
\hline 1 & $\sin 2 \Psi_{1} \cos 2 \theta_{1}+\sin 2 \Psi_{2} \cos 2 \theta_{2}$ & $\begin{aligned} \sin 2 \Psi_{1} \cos 2 \Psi_{2} \sin 2 \theta_{1} & \\
& +\sin 2 \Psi_{2} \cos 2 \Psi_{1} \sin 2 \theta_{2}\end{aligned}$ \\
\hline 2 & $\sin 2 \Psi_{1} \sin 2 \theta_{1}-\sin 2 \Psi_{2} \sin 2 \theta_{2}$ & $\cos 2 \Psi_{1} \sin 2 \Psi_{2} \cos 2 \theta_{2}$ \\
\hline 3 & $2 \cos \left(\Psi_{1}+\Psi_{2}\right) \cos \left(\Psi_{1}-\Psi_{2}\right)$ & $-\sin 2 \Psi_{1} \sin 2 \Psi_{2} \sin 2\left(\theta_{1}+\theta_{2}\right)$ \\
\hline
\end{tabular}

c'est-à-dire par exemple si $\Psi_{1}=0$ et $\Psi_{2}=\pi / 4$ (ou

\begin{tabular}{|c|c|c|c|c|c|}
\hline & & & \multicolumn{2}{|c|}{ Polariseur LINÉAIRE } & \multirow[b]{2}{*}{$\begin{array}{l}\text { Polariseur } \\
\text { CIRGULAIRE }\end{array}$} \\
\hline & $\mathrm{DE}$ & $\begin{array}{l}\text { PAS } \\
\text { POLARISEUR }\end{array}$ & $\begin{array}{l}\text { Parallèle } \\
\text { A OX ou OY }\end{array}$ & $\begin{array}{c}\mathrm{A} 45^{\circ} \\
\mathrm{DE} \text { OX } \mathrm{ET} \text { OY }\end{array}$ & \\
\hline$R_{0}$ & & 2 & 2 & 2 & 2 \\
\hline$R_{1}$ & & 0 & 0 & \pm 2 & 0 \\
\hline$R_{2}$ & & 0 & 0 & 0 & \pm 2 \\
\hline$R_{3}$ & & 0 & \pm 2 & 0 & 0 \\
\hline
\end{tabular}
vice versa), ce qui correspond à un polariseur linéaire et un analyseur circulaire (ou vice versa).

\section{TABLEAU I}

TABLEAU II 


\section{TABLEAU III}

\begin{tabular}{|c|c|c|c|c|}
\hline & \multirow[b]{2}{*}{$\begin{array}{c}\text { PAS } \\
\text { D'ANALYSEUR }\end{array}$} & \multirow{2}{*}{$\begin{array}{c}\text { POLARISEUR } \\
\text { ET ANALYSEUR } \\
\text { LINÉAIRES } \\
\text { A } 45^{\circ} \\
\text { L'UN DE L'AUTRE }\end{array}$} & \multicolumn{2}{|c|}{ ANALYSEUR GIRGULAIRE } \\
\hline & & & $\begin{array}{l}\text { Polariseur } \\
\text { PARALlÈlE } \\
\text { A OX OU OY }\end{array}$ & $\begin{array}{c}\text { Polariseur } \\
\text { A } 45^{\circ} \\
\text { DE OX Et OY }\end{array}$ \\
\hline$V_{0}$ & 0 & 0 & 0 & 0 \\
\hline$V_{1}$ & 0 & 0 & \pm 1 & 0 \\
\hline$V_{2}$ & 0 & \pm 1 & 0 & 0 \\
\hline$V_{3}$ & 0 & 0 & 0 & \pm 1 \\
\hline
\end{tabular}

$V_{2}$ est maximum si $\theta_{1}=\theta_{2}=0, \Psi_{1}-\Psi_{2}=\pi / 4$, ce qui correspond à deux polarisations linéaires faisant entre elles un angle de $45^{\circ}$.

$V_{3}$ est maximum si $\theta_{1}+\theta_{2}=\pi / 4, \Psi_{1}=\Psi_{2}=\pi / 4$, c'est-à-dire par exemple si $\theta_{1}=0$ et $\theta_{2}=\pi / 4$ (ou vice versa); on a alors un polariseur linéaire et un analyseur circulaire (ou vice versa).

Les résultats correspondants sont rassemblés sur le tableau III.

Nous sommes conduits à étudier particulièrement deux cas :

a) Lorsque le polariseur et l'analyseur sont linéaires $\left(\theta_{1}=\theta_{2}=0\right)$, le signal de détection optique s'écrit :

$$
\begin{aligned}
\Delta I & =\alpha \Delta E^{\prime} I_{0} b_{2}(t) \sin \left(2 \Psi_{2}-2 \Psi_{1}\right) \\
& =\frac{\alpha \Delta E^{\prime}}{\sqrt{2}} I_{0} c^{(1)}\left\langle I_{Z}\right\rangle \sin \left(2 \Psi_{2}-2 \Psi_{1}\right) .
\end{aligned}
$$

Le signal n'est sensible qu'à l'orientation $\left\langle I_{Z}\right\rangle$, qui correspond à une biréfringence circulaire de la vapeur, et donc à une rotation du plan de la polarisation lumineuse (effet Faraday).

b) Lorsque le polariseur est linéaire et l'analyseur circulaire (ou vice versa), on a $\theta_{1}=0, \theta_{2}=\Psi_{2}=\pi / 4$, et le signal $\Delta I$, qui ne dépend que de l'alignement dans l'état fondamental, s'écrit :

$\Delta I=\alpha \Delta E^{\prime} I_{0}\left[b_{1}(t) \cos 2 \Psi_{1}-b_{3}(t) \sin 2 \Psi_{1}\right]$.

L'étude de $\Delta I$ en fonction de $\Psi_{1}$ permet donc, comme plus haut, de déterminer les quantités $\left\langle I_{X}^{2}-I_{Y}^{2}\right\rangle$ et $\left\langle I_{X} I_{Y}+I_{X} I_{Y}\right\rangle$.

Nous verrons plus loin que l'alignement correspond à une biréfringence linéaire de la vapeur; cette dernière transforme donc la polarisation lumineuse linéaire incidente en une polarisation transmise qui, en général, est elliptique. On comprend donc le rôle de l'analyseur, qui permet de détecter le degré de polarisation circulaire de la lumière ainsi obtenue.

3. Comparaison des deUX mÉTHOdes. - Comparons les signaux de détection optique obtenus soit avec un faisceau résonnant, soit avec un faisceau non résonnant. a) De manière générale, nous avons vu qu'avec un faisceau non résonnant on parvient, mieux qu'avec un faisceau résonnant, à isoler les différentes grandeurs $b_{n}(t)$ dans le signal de détection optique.

$b$ ) Les amplitudes des signaux obtenus dans les deux cas sont comparables. Supposons en effet que l'on s'intéresse à la grandeur $b_{n}(t)$; on choisit les polariseurs et analyseurs les mieux adaptés pour détecter cette grandeur, soit avec un faisceau résonnant (on a alors $R_{n}= \pm 2$ ), soit avec un faisceau non résonnant (on a alors $V_{n}= \pm 1$ ). Les variations relatives du signal de détection optique sont, dans chacun des deux cas :

$$
\left\{\begin{array}{l}
\frac{\Delta I_{1}}{I_{0}}=\alpha \Gamma^{\prime} b_{n}(t)+\ldots \\
\frac{\Delta I_{2}}{I_{0}}=\alpha \Delta E^{\prime} b_{n}(t)+\ldots
\end{array}\right.
$$

Rappelons que $\alpha$ est un nombre inversement proportionnel à $I_{0}$. Les variations relatives du signal de détection optique, proportionnelles soit à $\Gamma^{\prime} / I_{0}$, soit à $\Delta E^{\prime} \mid I_{0}$, sont donc comparables; en effet, ces deux rapports (qui ne dépendent que de la composition spectrale du faisceau lumineux et non de son intensité totale) peuvent avoir en pratique des valeurs qui sont approximativement du même ordre de grandeur.

c) Un faisceau non résonnant permet, dans certains cas, d'utiliser des épaisseurs optiques non faibles, ce qui permet d'obtenir des signaux de détection optique plus grands [27], [28].

d) La perturbation apportée au système atomique par un faisceau non résonnant est souvent moins gênante que celle d'un faisceau résonnant. Par exemple, certaines mesures de temps de relaxation atomique sont plus aisées avec un faisceau non résonnant [19], [20], [29], [30].

On peut donc dire que, dans les cas où elle peut être utilisée, la détection optique avec un faisceau non résonnant semble présenter le plus grand nombre d'avantages.

C. Etude des signaux lumineux modulés. - Nous avons vu plus haut que les variations dans le temps 
du signal optique $\Delta I$ sont données par les variations des nombres $b_{n}(t)$, définis en (I.A.10). Les valeurs moyennes dont dépendent ces nombres peuvent faire intervenir les populations $\sigma_{\mu \mu}$ de la matrice densité $\sigma_{f}(t)$, comme ses « cohérences hertziennes " $\sigma_{\mu \mu^{\prime}}$. Nous supposons que les atomes sont placés dans un champ magnétique statique $\mathbf{H}_{0}$ et un champ de radiofréquence $\mathbf{H}_{\mathbf{1}}(t)$ tournant à la fréquence $\omega / 2 \pi$ autour de $\mathbf{H}_{0}$, et qu'ils sont soumis à l'action d'un faisceau lumineux de pompage de polarisation « non cohérente »[2]. Dans ces conditions, on sait que les cohérences hertziennes $\sigma_{\mu \mu^{\prime}}$ sont des fonctions sinusoïdales du temps, de pulsation $\left(\mu^{\prime}-\mu\right) \omega$. Le signal de détection optique comprend donc en général des modulations ayant ces pulsations. Dans ce paragraphe, nous allons étudier ces modulations, ainsi que la manière de les détecter dans deux cas particuliers : le faisceau détecteur se propage parallèlement au champ magnétique $\mathbf{H}_{0}$, ou perpendiculairement.

1. Faisceau Se propageant parallèlement a $\mathbf{H}_{0}$. Dans ce cas, on voit sur les équations (I.A.10) que $b_{0}(t)$ et $b_{2}(t)$ sont constants, alors que $b_{1}(t)$ et $b_{3}(t)$ sont modulés à la pulsation $2 \omega$.

On peut poser, avec un choix convenable de l'origine des temps :

$$
\left\{\begin{array}{l}
b_{1}(t)=\beta \sin 2 \omega t \\
b_{3}(t)=\beta \cos 2 \omega t .
\end{array}\right.
$$

a) Faisceau résonnant. - On voit sur (I.B.2) que la partie modulée du signal de détection optique s'écrit, lorsque l'on utilise un polariseur linéaire :

$$
\Delta I_{m}=-\alpha \Gamma^{\prime} I_{0} \beta \cos \left(2 \omega t-2 \Psi_{1}\right) .
$$

L'amplitude de la modulation lumineuse obtenue est donc indépendante de $\Psi_{1}$; sa phase varie comme $2 \Psi_{1}$.

b) Faisceau non résonnant. - On utilise un polariseur linéaire et un analyseur circulaire; d'après l'équation (I.B.5), le signal de détection optique s'écrit alors :

$$
\Delta I=\alpha \Delta E^{\prime} I_{0} \beta \sin \left(2 \omega t-2 \Psi_{1}\right)
$$

La modulation lumineuse a encore une amplitude indépendante de $\Psi_{1}$, et une phase qui varie comme $2 \Psi_{1}$; elle est en quadrature avec la modulation observée avec un faisceau résonnant.

\section{Faisceau Se propageant perpendiculairement} A $\mathbf{H}_{0}$. - Nous supposons que $\mathbf{H}_{0}$ est parallèle à l'axe OX (voir fig. 2); il est commode d'introduire un trièdre Oxyz dont l'axe $\mathrm{Oz}$ porte le champ magnétique $\mathbf{H}_{0}$, afin d'étudier l'évolution dans le temps des diverses grandeurs $b_{n}(t)$. En utilisant (I.A.10) et (I.A.13), il vient :

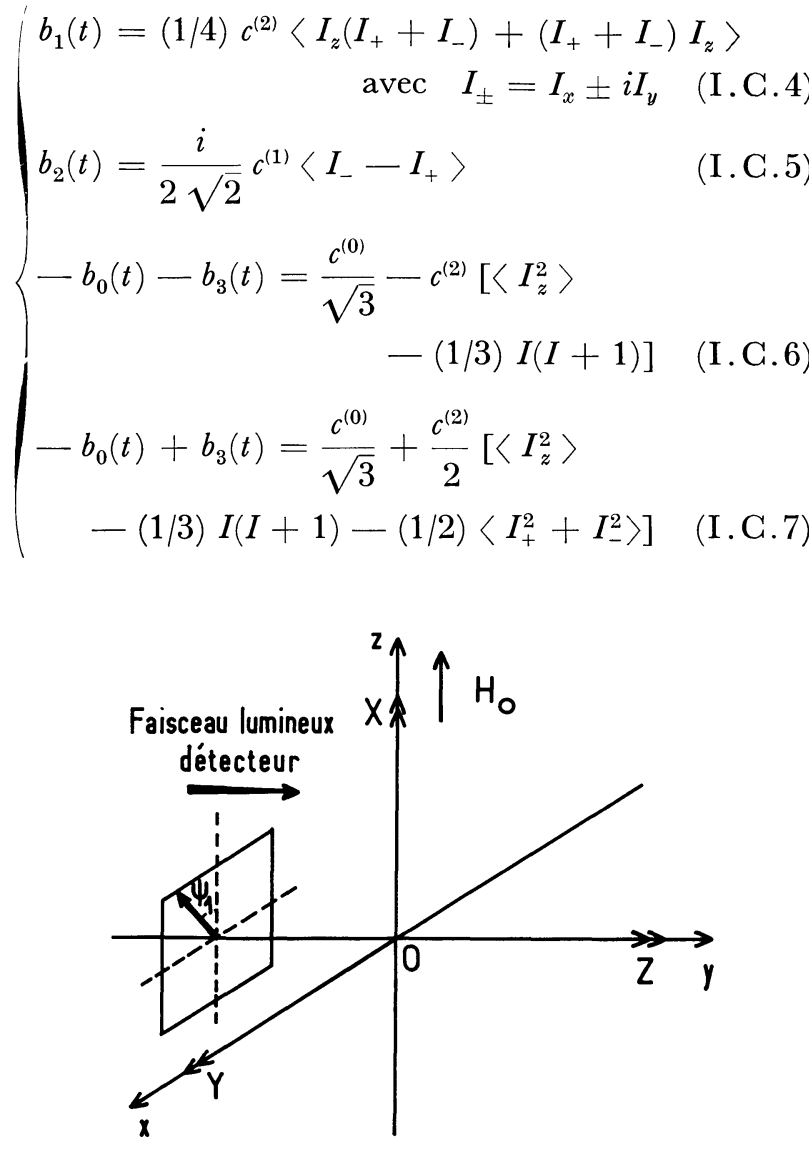

FIG. 2.

$b_{1}(t)$ et $b_{2}(t)$ évoluent donc à la pulsation $\omega,-b_{0}(t)$ $-b_{3}(t)$ est constant, et $-b_{0}(t)+b_{3}(t)$ comprend une partie constante ainsi qu'une partie évoluant à la pulsation $2 \omega$. Comme plus haut, nous allons distinguer deux cas :

a) Faisceau résonnant. - Rappelons que (cf. tableau II), sans polariseur ni analyseur, on observe la grandeur $b_{0}(t)$; le signal optique comprend donc dans ce cas une modulation à la pulsation $2 \omega$, qui correspond à l'alignement dans la direction $\mathrm{OZ}$.

Avec un polariseur circulaire, on détecte simultanément les grandeurs $b_{0}(t)$ et $b_{2}(t)$ (cf. tableau II); à la modulation à $2 \omega$ déjà signalée s'ajoute une modulation à $\omega$ due à l'orientation.

Avec un polariseur linéaire, on ne détecte que l'alignement (cf. éq. (I.B.2)). Il apparaît donc deux modulations lumineuses : l'une, de pulsation $\omega$, correspond à $b_{1}(t)$ et est proportionnelle à $R_{1} \propto \sin 2 \Psi_{1}^{\circ}$; l'autre, de pulsation $2 \omega$, correspond à $-b_{0}(t)+b_{3}(t)$ et est proportionnelle à $-R_{0}+R_{3} \propto \sin ^{2} \Psi_{1}$. On retrouve bien, en faisant $\Psi_{1}=0$, le fait que lorsque la polarisation $\left|\mathbf{e}_{\lambda_{0}}\right\rangle$ n'est pas cohérente, le signal ne peut contenir aucune modulation lumineuse. 
b) Faisceau non résonnant. - Avec un polariseur et un analyseur linéaires, on détecte la grandeur $b_{2}(t)$ (voir éq. (I.B.4)) et donc une modulation de pulsation $\omega$, proportionnelle à $\sin \left(2 \Psi_{2}-2 \Psi_{1}\right)$, qui correspond à l'orientation $\left\langle I_{Z}\right\rangle$.

Avec un polariseur linéaire et un analyseur circulaire (ou vice versa), le signal de détection optique ne dépend que de $b_{1}(t)$ et $b_{3}(t)$ (voir éq. (I.B.5)). Il apparaît donc deux modulations lumineuses dues à l'alignement : la première, qui correspond à $b_{1}(t)$, a la pulsation $\omega$ et est proportionnelle à $\cos 2 \Psi_{1}$; la seconde, qui correspond à $b_{3}(t)$, a la pulsation $2 \omega$ et est proportionnelle à $\sin 2 \Psi_{1}^{\circ}$.

Remarquons que, lorsque le faisceau lumineux détecteur se propage perpendiculairement à $\mathbf{H}_{0}$, ce sont les amplitudes des modulations, et non leurs phases, qui varient en fonction du polariseur ou de l'analyseur; par contre, lorsque le faisceau se propage parallèlement à $\mathbf{H}_{\mathbf{0}}$, on se trouve dans la situation contraire.

Signalons enfin que les résultats de ce paragraphe ont été vérifiés expérimentalement, et que l'accord entre la théorie et l'expérience est satisfaisant [26].

D. Influence de la composition spectrale du faisceau détecteur; cas des isotopes impairs du mercure. 1. Position du PROBLÈme, DÉfinitions. - Dans l'équation (I.A.15), l'influence sur le signal optique $\Delta I$ de la composition spectrale du faisceau lumineux détecteur est contenue dans les facteurs $\alpha \Gamma^{\prime}$ et $\alpha \Delta E^{\prime}$. Rappelons toutefois que pour obtenir cette équation, nous n'avons considéré dans l'état excité qu'un ensemble de sous-niveaux $|F, m\rangle$, issus d'un seul niveau de moment cinétique total $F$. Cette simplification revient dans le cas des isotopes impairs du mercure à supposer, comme dans JP1 et JP2, que le faisceau lumineux détecteur ne peut faire subir aux atomes de la vapeur que des transitions vers un seul niveau hyperfin de l'état excité. Or cette hypothèse n'est pas toujours réalisée, notamment en ce qui concerne les transitions virtuelles.

Nous allons donc dans ce paragraphe tenir compte de l'influence de la structure hyperfine dans l'état excité $6^{3} P_{1}$ (ou $6^{1} P_{1}$ ) du mercure. Les différences d'énergie Zeeman à l'intérieur du sous-niveau hyperfin $F$ entre les états $|F, m\rangle$ et $\left|F, m^{\prime}\right\rangle$ sont encore supposées négligeables devant la largeur $\hbar \Delta$ de la raie excitatrice (ou la largeur Doppler $\hbar \Delta^{\prime}$ de la raie d'absorption de la vapeur). Par contre, nous ne faisons aucune hypothèse concernant les différences d'énergie entre les états $|F m\rangle$ et $\left|F^{\prime} m^{\prime}\right\rangle\left(F \neq F^{\prime}\right)$. Dans ces conditions, on montre à partir des résultats de JP3 qu'il faut remplacer l'équation (I.A.1) par :

$\pi_{T}-\pi_{I}=-\alpha \sum_{F}\left\{\frac{\Gamma_{F}^{\prime}}{2}\left[B_{F}(t), \pi_{I}\right]_{+}+i \Delta E_{F}^{\prime}\left[B_{F}(t), \pi_{I}\right]\right\}$.

Dans cette expression, $\Gamma_{F}^{\prime}$ et $\Delta E_{F}^{\prime}$ sont obtenus par généralisation des nombres $\Gamma^{\prime}$ et $\Delta E^{\prime}$, dont la définition est rappelée en (I.A.3); ils correspondent à la contribution de la composante hyperfine $F$ à l'élargissement et au déplacement de l'état fondamental sous l'effet du faisceau lumineux détecteur. Leur définition est donnée par :

$$
\begin{gathered}
\frac{\Gamma_{F}^{\prime}}{2}+i \Delta E_{F}^{\prime}=\frac{i q^{2}}{\hbar^{2}} \frac{|\langle J=1\|\mathbf{S}\| J=0\rangle|^{2}}{3} \\
\int I(\omega) \mathrm{d} \omega \int \frac{f(\mathbf{v}) \mathrm{d}^{3} \mathbf{v}}{\omega-\omega_{0}^{H^{\prime}}-\mathbf{k} \cdot \mathbf{v}+i \Gamma / 2} .
\end{gathered}
$$

Les seules notations qui ne soient pas déjà introduites dans JP4 sont :

- $\hbar \omega_{0}^{F}$, différence d'énergie entre le sous-niveau hyperfin $F$ et le niveau fondamental. La dépendance en $F$ de $\Gamma_{F}^{\prime}$ et $\Delta E_{F}^{\prime}$ provient uniquement, dans l'équation (I.D.2), de $\omega_{0}^{F}$. Lorsque l'on a :

$$
\Delta\left(\text { ou } \Delta^{\prime}\right) \gg\left|\omega_{0}^{F}-\omega_{0}^{F^{\prime \prime}}\right|
$$

pour $F$ et $F^{\prime}$ quelconques (c'est-à-dire lorsque la structure hyperfine de la transition optique utilisée n'est pas résolue dans la largeur de la raie excitatrice : illumination «broad line »), $\Gamma_{F}^{\prime}$ et $\Delta E_{F}^{\prime}$ sont indépendants de $F$.

$-q^{2}|\langle J=1\|\mathbf{S}\| J=0\rangle|^{2}$, carré du module de l'élément de matrice réduit du dipôle électrique $q \mathbf{S}$ entre les états purement électroniques $\left|J=1, m_{J}\right\rangle$ dont sont issus les divers sous-niveaux excités $|F, m\rangle$, et l'état fondamental $\left|J=0, m_{J}=0\right\rangle$. Nous avons utilisé le fait que l'opérateur $q \mathbf{S}$ n'agit pas sur le spin nucléaire I; cette remarque va aussi nous permettre de définir les éléments de matrice des composantes standard de $\mathbf{D}$ (partie angulaire de $q \mathbf{S}$ ) par :

$$
\left\{\begin{aligned}
\left\langle F, m\left|D_{q}\right| I, \mu\right\rangle= & \sum_{m_{I} m_{J}}\left\langle F m \mid I 1 m_{I} m_{J}\right\rangle \\
& \left\langle 010 q \mid 1 m_{J}\right\rangle\left\langle I 0 m_{I} 0 \mid I \mu\right\rangle \\
= & \langle F m \mid I 1 \mu q\rangle \\
\left\langle I, \mu\left|D_{q}\right| F, m\right\rangle= & (-1)^{q}\left\langle F, m\left|D_{-q}\right| I, \mu\right\rangle .
\end{aligned}\right.
$$

Remarquons que dans l'équation (I.D.1) apparaît une matrice $B_{F}(t)$ différente pour chaque niveau $F$; l'expression de $B_{F}(t)$ est celle qui est donnée en (I.A.2), où $P_{e}$ a été remplacé par :

$$
P_{F}=\sum_{-F \leqslant m \leqslant+F}|F, m\rangle\langle F, m|
$$

projecteur sur le sous-niveau hyperfin $F$.

Comme plus haut, nous allons décomposer chaque matrice $B_{F}(t)$ sur les matrices de Pauli, en posant :

$$
B_{F}(t)=\sum_{n=0}^{3} b_{n}^{F}(t) \sigma_{n}
$$

ce qui nous permet d'obtenir la relation :

$\Delta I=-\alpha I_{0} \sum_{F, n} b_{n}^{F}(t)\left[\frac{\Gamma_{F}^{\prime}}{2} R_{n}-\Delta E_{F}^{\prime} V_{n}\right]$. 
Cette équation est la généralisation de (I.A.15) lorsque l'on tient compte de la structure hyperfine du niveau excité.

Les nombres $b_{n}^{F}(t)$ peuvent s'exprimer comme en (I.A.10), en fonction des valeurs moyennes dans l'état fondamental d'opérateurs $T_{q}^{(k)}(F)$, qui maintenant dépendent de $F$, et sont obtenus en remplaçant dans (I.A.11) $P_{e}$ par $P_{F}$.

Notons que, d'après le théorème de Wigner-Eckart, deux opérateurs $T_{q}^{(k)}(F)$ qui ne diffèrent que par la valeur de $F$ sont proportionnels. Il est commode d'introduire, comme en (I.A.12) et (I.A.13), les coefficients de proportionnalité $c^{(k)}(F)$, dépendant de $F$, entre chaque opérateur $T_{q}^{(k)}(F)$ et l'un des 5 opérateurs tensoriels : $1, I_{Z}, 3 I_{Z}^{2},-I(I+1)$ et $\left(I_{X} \pm i I_{Y}\right)^{2}$. On peut alors exprimer le signal $\Delta I$ en fonction des mêmes grandeurs dans l'état fondamental qu'au paragraphe $\mathrm{A}$; la seule différence est que, pour tenir compte de l'existence des différents niveaux $F$, il faut remplacer, dans la contribution de chaque observable d'ordre $k, \Gamma^{\prime}$ par $\sum_{F} c^{(k)}(F) \Gamma_{F}^{\prime}$ et $\Delta E^{\prime}$ par $\sum_{F} c^{(k)}(F) \Delta E_{F}^{\prime}$.
Notons toutefois que les coefficients $R_{n}$ et $V_{n}$, qui ne dépendent pas de $F$, restent inchangés. Il en résulte que, tant que l'on ne compare pas entre eux des signaux relatifs à des observables d'ordres $k$ différents, et dus à des transitions de nature différente (réelles ou virtuelles), on aboutit aux mêmes conclusions qu'aux paragraphes $B$ et $\mathrm{C}$; les résultats énoncés dans ces paragraphes sont donc encore valables lorsque l'on tient compte de la structure hyperfine du niveau excité.

Par contre, les variations du signal $\Delta I$, donné par l'équation (I.D.5), en fonction de la composition spectrale de la lumière excitatrice, sont maintenant complexes : elles dépendent de l'ordre de l'observable étudiée, et font intervenir les coefficients $c^{(k)}(F)$, dont nous allons calculer les variations en fonction de $k$ et de $F$. En reportant les relations (I.D.3) dans l'expression des éléments de matrice de $T_{q}^{(k)}(F)$, on obtient (en remplaçant les coefficients de Glebsch-Gordan par des coefficients $3 j$, de manière à utiliser leurs relations de somme [31]):

$$
\begin{aligned}
\left\langle\mu^{\prime}\left|T_{q}^{(k)}(F)\right| \mu^{\prime \prime}\right\rangle= & (-1)^{q}(2 F+1) \sqrt{2 k+1} \\
& \times \sum_{m, q^{\prime}+q^{\prime \prime}=q}(-1)^{q^{\prime}}\left(\begin{array}{ccc}
1 & 1 & k \\
q^{\prime} & q^{\prime \prime} & -q
\end{array}\right)\left(\begin{array}{ccc}
1 & F & I \\
-q^{\prime \prime} & m & -\mu^{\prime \prime}
\end{array}\right)\left(\begin{array}{ccc}
F & 1 & I \\
-m & -q^{\prime} & \mu^{\prime}
\end{array}\right) \\
= & (-1)^{I+F+k} \frac{(2 F+1) \sqrt{2 k+1}}{\sqrt{2 I+1}}\left\{\begin{array}{lll}
I & I & k \\
1 & 1 & F
\end{array}\right\}\left\langle I k \mu^{\prime \prime} q \mid I \mu^{\prime}\right\rangle .
\end{aligned}
$$

Cette relation permet de calculer les coefficients $c^{(k)}(\boldsymbol{F})$. On a alors :

$$
\sum_{F} c^{(k)}(F) \Gamma_{F}^{\prime} \propto \sum_{F}(-1)^{I+F}(2 F+1)\left\{\begin{array}{lll}
I & I & k \\
1 & 1 & F
\end{array}\right\} \Gamma_{F}^{\prime} .
$$

Cette expression donne, dans le cas d'un faisceau résonnant, les variations du signal optique correspondant à une observable d'ordre $k$ en fonction de la composition spectrale du faisceau détecteur. Pour un faisceau non résonnant, il suffit de remplacer $\Gamma_{F}^{\prime}$ par $\Delta E_{F}^{\prime}$. La formule (I.D.7) va nous permettre d'étudier quelques cas particuliers.

2. CAS DE ${ }^{199} \mathrm{Hg}: I=1 / 2$. - Le niveau excité, pour lequel $J$, moment angulaire électronique, vaut 1 , comprend deux sous-niveaux hyperfins $F=1 / 2$ et $F=3 / 2$. On montre, en reportant ces valeurs de $I, J$ et $F$ dans (I.D.7), que les signaux correspondant à la population totale de l'état fondamental sont proportionnels à $\left(\Gamma_{1 / 2}^{\prime}+2 \Gamma_{3 / 2}^{\prime}\right)$. Dans le cas de l'orientation, les signaux sont proportionnels à $\left(\Gamma_{1 / 2}^{\prime}-\Gamma_{3 / 2}^{\prime}\right)$ pour un faisceau résonnant, à $\left(\Delta E_{1 / 2}^{\prime}-\Delta E_{3 / 2}^{\prime}\right)$ pour un faisceau non résonnant.

Nous retrouvons bien que l'absorption lumineuse isotrope correspondant à la population totale de l'état fondamental est, pour chaque composante hyperfine $F$, proportionnelle au poids statistique $(2 F+1)$ du niveau $F$. On remarque de plus que les signaux dus à l'orientation sont nuls dans le cas d'une illumination « broad-line », c'est-à-dire par exemple si :

$$
\Delta \gg\left|\omega_{0}^{1 / 2}-\omega_{0}^{3 / 2}\right| .
$$

Ce résultat peut s'interpréter de la manière suivante : on montre dans JP1 et JP2 que les phénomènes transitoires dus au processus d'absorption d'un photon par un atome durent un temps $1 / \Delta$; la condition $\Delta \gg\left|\omega_{0}^{1 / 2}-\omega_{0}^{3 / 2}\right|$ signifie donc qu'au cours de ce processus le couplage hyperfin entre $\mathbf{I}$ et $\mathbf{J}$ dans l'état excité n'a pas le temps de se produire. On comprend alors pourquoi l'absorption lumineuse, qui ne fait intervenir aucun couplage direct entre I et l'onde lumineuse, ne peut être sensible à l'orientation de I dans l'état fondamental.

3. CAS DE ${ }^{201} \mathrm{Hg}: I=3 / 2$. - Le niveau excité comprend trois sous-niveaux hyperfins, $F=1 / 2,3 / 2$ et $5 / 2$. On montre comme plus haut que les signaux optiques correspondant à la population totale de l'état fondamental sont proportionnels à :

$$
2 \Gamma_{1 / 2}^{\prime}+4 \Gamma_{3 / 2}^{\prime}+6 \Gamma_{5 / 2}^{\prime} \text {. }
$$


Dans le cas de l'orientation, on obtient :

$5 \Gamma_{1 / 2}^{\prime}+4 \Gamma_{3 / 2}^{\prime}-9 \Gamma_{5 / 2}^{\prime}$ $5 \Delta E_{1 / 2}^{\prime}+4 \Delta E_{3 / 2}^{\prime}-9 \Delta E_{5 / 2}^{\prime} \quad$ (transitions virtuelles)

et dans le cas de l'alignement :

$-5 \Gamma_{1 / 2}^{\prime}+8 \Gamma_{3 / 2}^{\prime}-3 \Gamma_{5 / 2}^{\prime}$ $-5 \Delta E_{1 / 2}^{\prime}+8 \Delta E_{3 / 2}^{\prime}-3 \Delta E_{5 / 2}^{\prime}$ (transitions virtuelles) $\}$.

Les signaux correspondant à la population totale font ici encore intervenir les poids statistiques des niveaux $F$; ceux qui sont dus à l'orientation ou à l'alignement sont nuls pour une illumination « broad line ». Les interprétations de ces résultats sont les mêmes que dans le cas de ${ }^{199} \mathrm{Hg}$.

Les coïncidences hyperfines de la raie $\lambda=2537 \AA$ du mercure permettent, en changeant l'isotope contenu dans la lampe dont est issu le faisceau détecteur, de faire varier les grandeurs relatives des divers nombres $\Gamma_{F}^{\prime}$ et $\Delta E_{F}^{\prime}$. Les formules qui viennent d'être données permettent donc de choisir l'isotope le plus approprié à la détection de chaque observable de l'état fondamental. Par exemple, dans le cas de l'alignement détecté avec un faisceau non résonnant, on voit sur (I.D.10) qu'il est préférable d'utiliser une raie lumineuse proche de la composante $F=3 / 2$ de ${ }^{201} \mathrm{Hg}$; si, de plus, la fréquence centrale du faisceau détecteur tombe entre les deux composantes $F=3 / 2$ et $F=5 / 2$ de ${ }^{201} \mathrm{Hg}$, distantes de quelques largeurs Doppler, $\Delta E_{3 / 2}^{\prime}$ et $\Delta E_{5 / 2}^{\prime}$ sont du même ordre de grandeur, mais de signes opposés. On voit alors sur (I.D.10) que les signaux dus aux deux composantes hyperfines s'ajoutent. Or ce cas correspond à l'utilisation d'une lampe remplie de ${ }^{196} \mathrm{Hg}$; c'est donc cet isotope qui est le meilleur pour détecter l'alignement avec un faisceau non résonnant.

Signalons enfin que nous avons vérifié les formules (I.D.9) et (I.D.10) en effectuant des expériences qui ont été décrites par ailleurs [26].

Remarque : Le calcul que nous venons de faire permet de tenir compte de la structure hyperfine des transitions optiques d'un atome quelconque pour lequel $J=0$ dans l'état fondamental $\left(^{6}\right)$. On peut transpo-

(6) La généralisation au cas où $J$ est quelconque dans l'état fondamental (ce qui est le cas, par exemple, pour les alcalins) est effectuée dans l'appendice I. ser ces résultats à l'étude de la structure fine, dans le cas des atomes pour lesquels $L=0$ dans l'état fondamental.

Étudions par exemple le cas de la raie $\lambda=10830 \AA$ de ${ }^{4} \mathrm{He}$, pour laquelle le niveau métastable $2^{3} S_{1}$ joue le rôle de l'état fondamental; le niveau excité $2^{3} P$ comprend trois niveaux de structure fine, repérés par les valeurs de $J=0,1$ ou 2 . On montrerait dans ce cas qu'avec un faisceau résonnant les signaux optiques correspondant à la population totale du niveau $2^{3} S_{1}$ sont proportionnels à $\Gamma_{0}^{\prime}+3 \Gamma_{1}^{\prime}+5 \Gamma_{2}^{\prime}$; pour l'orientation et l'alignement, on obtiendrait respectivement :

$$
\left(-2 \Gamma_{0}^{\prime}-3 \Gamma_{1}^{\prime}+5 \Gamma_{2}^{\prime}\right) \quad \text { et } \quad\left(2 \Gamma_{0}^{\prime}-3 \Gamma_{1}^{\prime}+\Gamma_{2}^{\prime}\right)
$$

avec un faisceau résonnant;

$$
\left(-2 \Delta E_{0}^{\prime}-3 \Delta E_{1}^{\prime}+5 \Delta E_{2}^{\prime}\right)
$$

et

$$
\left(2 \Delta E_{0}^{\prime}-3 \Delta E_{1}^{\prime}+\Delta E_{2}^{\prime}\right)
$$

avec un faisceau non résonnant. Nous retrouvons dans ce cas certains résultats de Pancharatnam [23].

\section{APPENDICE I}

Nous donnons dans cet appendice les formules qui permettent, dans le cas des alcalins, de calculer l'influence sur les signaux optiques de la composition spectrale du faisceau détecteur. Les notations et les équations de départ sont celles du paragraphe $\mathrm{V}$ de JP4.

Les éléments de matrice de $\mathbf{D}$ sont donnés par (cf. JP4 (V.5) et (V.6)) :

$$
\begin{gathered}
\left\langle\mathscr{F} m\left|D_{q}\right| F_{\mu}\right\rangle=\sum_{m_{I}, m_{J}, m_{J}^{\prime}}\left\langle\mathscr{F} m \mid I J_{e} m_{I} m_{J}\right\rangle \\
\left\langle J_{f} 1 m_{J}^{\prime} q \mid J_{e} m_{J}\right\rangle\left\langle I J_{f} m_{I} m_{J}^{\prime} \mid F \mu\right\rangle
\end{gathered}
$$

qui peut encore s'écrire :

$$
\begin{array}{r}
\left\langle\mathscr{F} m\left|D_{q}\right| F \mu\right\rangle=(-1)^{1+I+J_{f}+\mathscr{F}} \sqrt{\left(2 J_{e}+1\right)(2 F+1)} \\
\left\{\begin{array}{lll}
1 & F & \mathscr{F} \\
I & J_{e} & J_{f}
\end{array}\right\}\langle F 1 \mu q \mid \mathscr{F} m\rangle . \quad \text { (III.2) }
\end{array}
$$

En reportant cette équation dans la définition des opérateurs $T_{q}^{(k)}(\mathscr{F})$ (voir JP4 (V.13)), on obtient, tous calculs faits :

$$
\begin{array}{r}
\left\langle F_{2} \mu_{2}\left|T_{q}^{(k)}(\mathscr{F})\right| F_{1} \mu_{1}\right\rangle=(-1)^{k+F_{1}+\mathscr{F}}\left(2 J_{e}+1\right)(2 \mathscr{F}+1) \sqrt{(2 k+1)\left(2 F_{1}+1\right)} \\
\left\{\begin{array}{ccc}
F_{1} & F_{2} & k \\
1 & 1 & \mathscr{F}
\end{array}\right\}\left\{\begin{array}{lll}
1 & F_{1} & \mathscr{F} \\
I & J_{e} & J_{f}
\end{array}\right\}\left\{\begin{array}{ccc}
1 & F_{2} & \mathscr{F} \\
I & J_{e} & J_{f}
\end{array}\right\}\left\langle F_{1} k \mu_{1} q \mid F_{2} \mu_{2}\right\rangle .
\end{array}
$$


Reportons ce résultat dans les définitions de $B_{F \mathscr{F}}(t)$ et $C_{\mathscr{F}}(t)$, en utilisant les résultats (III.13) et (III.14) de JP4; l'équation (V.11) permet alors de calculer la contribution de chaque grandeur atomique $T_{q}^{(k)}(\mathscr{F})$ au signal optique. Nous allons donner deux exemples :

a) Considérons la grandeur $\left\langle P_{F} T_{q}^{(k)} P_{F}\right\rangle$ (population, orientation ou alignement à l'intérieur du sous-niveau hyperfin $F$, ne dépendant que des « populations » et des « cohérences Zeeman »de $\sigma_{f}$ ). Les signaux optiques correspondants obtenus avec un faisceau résonnant $\left(\Delta E_{F \mathscr{F}}^{\prime} \ll \Gamma_{F \mathscr{F}}^{\prime}\right)$ sont proportionnels à :

$$
\begin{aligned}
\sum_{\mathscr{F}}(-1)^{F+\mathscr{F}}(2 \mathscr{F}+1) & \left\{\begin{array}{ccc}
F & F & k \\
1 & 1 & \mathscr{F}
\end{array}\right\} \\
& \left\{\begin{array}{lll}
1 & F & \mathscr{F} \\
I & J_{e} & J_{f}
\end{array}\right\}^{2} \Gamma_{F \mathscr{F}}^{\prime} .
\end{aligned}
$$

Cette formule est la généralisation au cas des alcalins de (I.D.7). Dans le cas d'un faisceau non résonnant, il suffit de remplacer $\Gamma_{F \mathscr{F}}^{\prime}$ par $\Delta E_{F \mathscr{F}}^{\prime}$.

Lorsque $\Gamma_{F \mathscr{F}}^{\prime}$ ne dépend pas de $\mathscr{F}$ (c'est-à-dire lorsque la structure hyperfine de l'état excité n'est pas résolue), l'expression (III.4) devient [32] :

$\sum_{\mathscr{F}}(-1)^{F+\mathscr{F}}(2 \mathscr{F}+1)\left\{\begin{array}{ccc}F & F & k \\ 1 & 1 & \mathscr{F}\end{array}\right\}\left\{\begin{array}{ccc}1 & F & \mathscr{F} \\ I & J_{e} & J_{f}\end{array}\right\}^{2} \Gamma_{F}^{\prime}$
$=(-1)^{k+F+I+J_{e}+2 J_{f}}\left\{\begin{array}{ccc}k & J_{f} & J_{f} \\ I & F & F\end{array}\right\}\left\{\begin{array}{ccc}k & J_{f} & J_{f} \\ J_{e} & 1 & 1\end{array}\right\} \Gamma_{F}^{\prime}$.
Les deux coefficients $6 j$ de cette expression sont nuls lorsque $k>2 J_{f}$. Nous retrouvons donc ici un résultat établi plus directement dans JP4 : on ne peut pas observer l'alignement $(k=2)$ dans l'état fondamental d'atomes alcalins $\left(J_{f}=1 / 2\right)$ lorsque la structure hyperfine dans l'état excité n'est pas résolue.

b) Considérons la grandeur $\left\langle P_{F_{2}} T_{q}^{(k)} P_{F_{1}}\right\rangle$ (partie de l'orientation ou de l'alignement qui dépend des « cohérences hyperfines » de $\sigma_{f}$ ). Nous supposons que le faisceau est résonnant et que les signaux optiques observés sont dus à l'absorption optique de la vapeur : nous négligeons donc dans (V.11) les termes en $\Delta E^{\prime}$ et ne conservons que l'anticommutateur qui contient $C_{\mathscr{F}}^{R}(t)$. Dans ces conditions, la contribution de $\left\langle P_{F_{2}} T_{q}^{(k)} P_{F_{1}}\right\rangle$ au signal de détection optique est proportionnelle à :

$\sum_{\mathscr{F}}(-1)^{F_{1}+\mathscr{F}}(2 \mathscr{F}+1)\left\{\begin{array}{ccc}F_{1} & F_{2} & k \\ 1 & 1 & \mathscr{F}\end{array}\right\}$

$\left\{\begin{array}{ccc}1 & F_{1} & \mathscr{F} \\ I & J_{e} & J_{f}\end{array}\right\}\left\{\begin{array}{ccc}1 & F_{2} & \mathscr{F} \\ I & J_{e} & J_{f}\end{array}\right\}\left(\Gamma_{F_{1} \mathscr{F}}^{\prime}+\Gamma_{F_{2} \mathscr{F}}^{\prime}\right)$.

Les signaux obtenus dans d'autres conditions que celles que nous avons supposées se déduisent aisément de l'équation (V.11) de JP4.

Signalons enfin que des calculs analogues ont été effectués par Happer et al. dans une étude des déplacements lumineux [2] d'un atome alcalin mis en présence d'un faisceau non résonnant [33].

\section{BIBLIOGRAPHIE}

[1] Cagnac (B.), Thèse, Paris, 1960 ; Ann. de Phys., 1961, 6, 467; J. Physique Rad., 1958, 19, 863.

[2] Cohen-Tannoudji (C.), Thèse, Paris, $1962 ;$ Ann. de Phys., 1961, 7, 423 et 469.

[3] Manuel (J.), Diplôme d'Études Supérieures, Paris, 1964.

[4] Dehmeit (H. G.), Phys. Rev., 1957, 105, 1924.

[5] Bell (W. E.) et Bloom (A. L.), Phys. Rev., 1957, 107, 1559.

[6] Bouchiat (M. A.), Thèse, Paris, 1964.

[7] Bouchiat (M. A.), J. Physique, 1965, 26, 415.

[8] Bouchiat (M. A.) et Grossetête (F.), J. Physique, 1966, 27, 353.

[9] Firester (A. H.) et Carver (T. R.), Phys. Rev. Letters, 1966, 17, 947 ; Phys. Rev., 1967, 164, 76.

[10] Mathur (B. S.), TANG (H.), Bulos (R.) et HaPPER (W.), Phys. Rev. Letters, 1968, 21, 1035.

[11] Colmgrove (F. D.) et Franken (P. A.), Phys. Rev., 1960, 119, 680 .

[12] Schearer (L. D.), Phys. Rev., 1962, 127, 512.
[13] Colfegrove (F. D.), Schearer (L. D.) et WaLTERS (G. K.), Phys. Rev., 1963, 132, 2561.

GreEnhow (R. C.), Phys. Rev., 1964, 136, 660.

[14] Partridge (R. B.) et Series (G. W.), Proc. Phys. Soc., $1966,88,983$ et 969.

[15] Donszelmann (A.), VAn Der Berg (C. J.) et VoeteLINK (P.), Phys. Letters, 1967, 26 A, 83.

[16] KASTLER (A.), J. Physique Rad., 1950, 11, 255 ; J. Opt. Soc. Am., 1957, 47, 460.

[17] Barrat (J. P.) et Cohen-Tannoudji (C.), J. Physique Rad., 1961, 22, 329 et 443.

[18] GozzinI (A.), C. R. Acad. Sc. (Fr.), 1962, 255, 1905.

[19] Manuel (J.) et Cohen-Tannoudji (C.), C. R. Acad. Sc., 1963, 257, 413; voir aussi [3].

[20] Strumia (F.), Nuovo Cimento, 1966, 44 B, 387, et 1967, $51 \mathrm{~B}, 82$.

[21] Novikov (L. N.), Zh. E.T.F., 1967, 6, 473, ou Soviet Physics (JETP), 1967, 6, 11 ; Optika i Spekt., 1968, 24, 866, ou Optics and Spect., 1968, 24, 465.

[22] Happer (W.) et Mathur (B. S.), Phys. Rev. Lett., 1967, 18, 577. 
[23] Pancharatnam (S.), J. Phys. B (Proc. Phys. Soc.), 1968, 1, 250.

[24] Pancharatnam (S.), Phys. Lett., 1968, 27 A, 509 ; Compte rendu de 1' "International Conference on Optical Pumping and Atomic Line Shape " (O.P.A.L.S.), Varsovie (Pologne).

[25] Cohen-Tannoudji (C.) et Lalö̈ (F.), J. Physique, 1967, 28, 505 et 722 .

[26] LaIö̈ (F.), LededC (M.) et Mrnguzzi (P.), C. $R$. Acad. Sc., 1968, 266 B, 1517, et C. R. Acad. Sc., 1968, 267 B, 328.

[27] AlzettTa (G.) et ARImondo (E.), Nuovo Cimento, à paraître.
[28] Izrä̈L (A.) et CaGnaC (B.), C. R. Acad. Sc., 1968, $267 \mathrm{~B}, 1207$.

IzRAËL (A.), Thèse $3^{\mathrm{e}}$ cycle, 1969, Paris.

[29] Cohen-Tannoudji (C.) et Brossel, (J.), C. R. Acad. Sc., 1964, 258 B, 6119.

[30] Minguzzi (P.), Strumia (F.) et Violino (P.), Nuovo Cimento, 1966, 46 B, 145.

[31] Voir par exemple Messiah (A.), "Mécanique quantique ", Dunod, Paris, 1963, p. 915, formule (33).

[32] Messiah (A.), op. cit., p. 915, formule (35).

[33] Happer (W.) et Mathur (B. S.), Phys. Rev., 1967, $163,12$.

Mathur (B. S.), TANG (H.) et Happer (W.), Phys. Rev., 1968, 171, 11. 